\title{
LP-HCLUS: a novel tool for the prediction of relationships between ncRNAs and human diseases
}

\author{
Emanuele Pio Barracchiaa ${ }^{1,2 \bowtie}$, Gianvito Pio ${ }^{1,2}$, Domenica D'Elia ${ }^{3}$, Michelangelo Ceci ${ }^{1,2,4}$ \\ 1 Department of Computer Science, University of Bari Aldo Moro, Bari, Italy \\ ${ }^{2}$ National Interuniversity Consortium for Informatics (CINI), Rome, Italy \\ ${ }^{3}$ Institute for Biomedical Technologies, National Research Council, Bari, Italy \\ ${ }^{4}$ Department of Knowledge Technologies, Jozef Stefan Institute, Ljubljana, Slovenia \\ Competing interests: EPB none; GP none; DD none; MC none
}

The discovery of a functional relationship between human diseases and non-coding RNAs (ncRNAs) is not new. In the last decade, it improved the elucidation of many diseases' mechanisms and the improvement of therapeutic approaches (Lekka and Hall, 2018; Wang et al., 2016; Yang et al., 2014). Nevertheless, the function of many ncRNAs is still unclear or completely unknown, and therefore, their role in human diseases is difficult, if not impossible, to be identified. We have developed a new system, called LP-HCLUS, that is able to predict previously unknown disease-ncRNA associations by exploiting multi-type hierarchical clustering techniques.

Differently from other approaches, LP-HCLUS is able to analyse and benefit from heterogeneous networks of interactions/relationships among multiple types of entities (e.g., diseases, ncRNAs, target genes) and relationships between them. To this aim, the proposed method first estimates the strength of the disease-ncRNA associations, exploiting both direct and indirect relationships. It constructs a hierarchy of heterogeneous clusters based on known and estimated relationships between diseases and ncRNAs. Finally, LP-HCLUS uses the generated clusters to induce new relationships, associating each of them with a certainty score. We conducted several experiments, comparing the performances achieved by LP-HCLUS with those obtained by two different competitors: HOCCLUS2 (Pio et al., 2013) and ncPred (Alaimo et al., 2014). In particular, we analysed two different datasets: HMDD v3.0, which contains data about relationships between diseases and miRNAs, and a dataset constructed integrating different

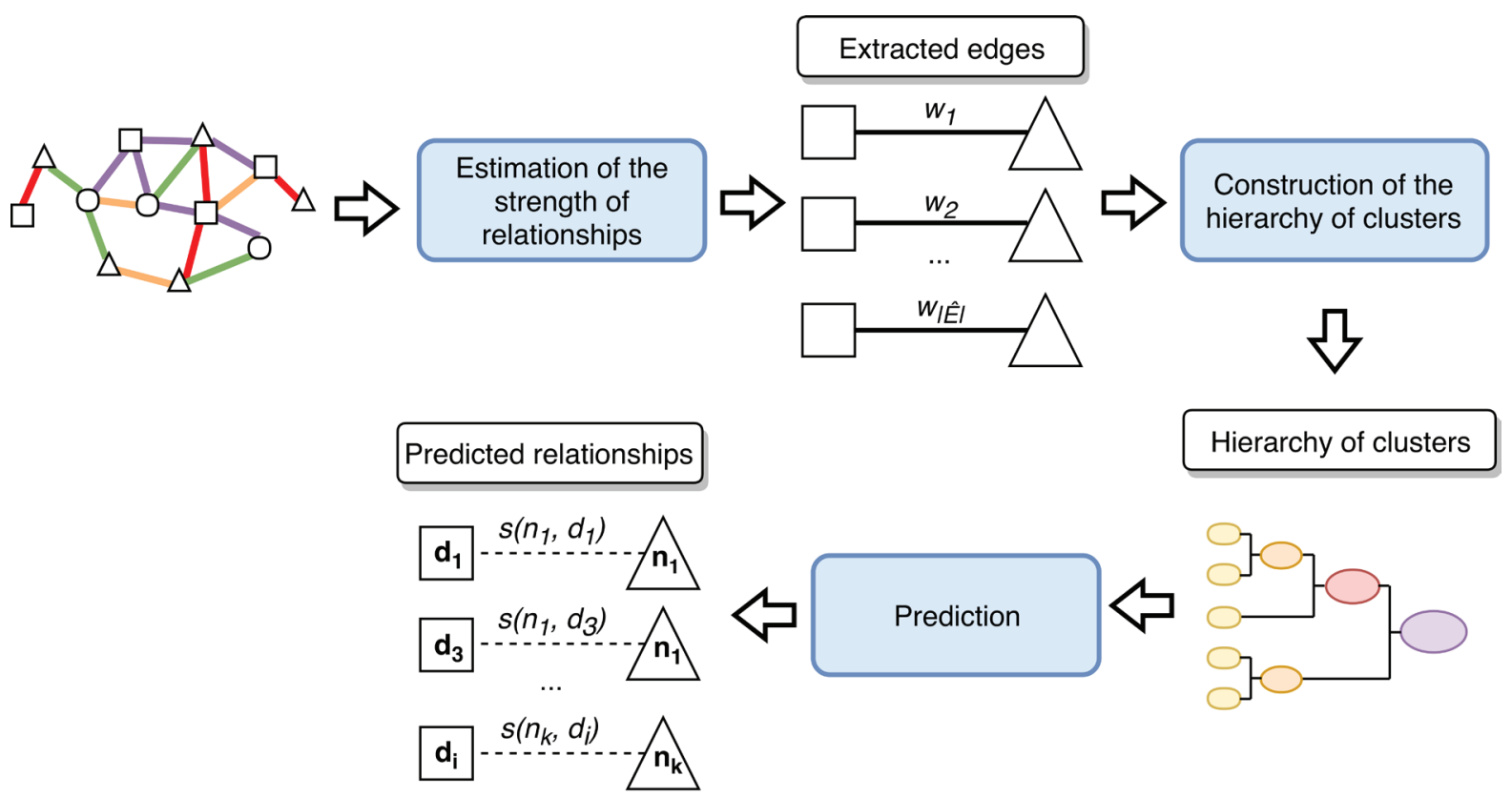

Figure 1. The workflow of the LP-HCLUS method.

(c) 2021 Barracchia et al.; the authors have retained copyright and granted the Journal right of first publication; the work has been simultaneously released under a Creative Commons Attribution Licence, which allows others to share the work, while acknowledging the original authorship and initial publication in this Journal. The full licence notice is available at http://journal.embnet.org. 
state-of-the-art data sources (Chen et al., 2013; Helwak et al., 2013; Bauer-Mehren et al., 2010; Jiang et al., 2009).

The results show that our system is able to outperform its competitors, and it can help biologists to conduct more focused research. Such a conclusion is also confirmed by a qualitative analysis conducted on the predicted associations that showed that many associations predicted by LP-HCLUS with a high certainty score have been subsequently validated and introduced in a more recent version of HMDD dataset (v3.2). The importance of such a development is also in its easy transfer for applications in any biological study involving heterogeneous data from different sources and types (e.g., different omics data, chemicals, biochemical and structural data, etc.).

\section{Availability of data and materials}

The system LP-HCLUS, the adopted datasets and all the results are available at: http://www.di.uniba. it/ gianvitopio/systems/lphclus/

\section{Acknowledgements}

We would like to acknowledge the financial support of the European Commission through the project MAESTRA - Learning from Massive, Incompletely annotated, and Structured Data (Grant Number ICT-2013-612944). We also acknowledge the financial support of the Ministry of Education, Universities and Research (MIUR) through the PON projects "Big Data Analytics" (AIM1852414 - Activity 1, Line 1) and TALIsMAn - Tecnologie di Assistenza personALizzata per il Miglioramento della quAlità della vitA (Grant N. ARS01_0111), and of
Italian National Research Council (CNR) through the InterOmics Flagship project.

\section{References}

1. Alaimo S, Giugno R, Pulvirenti A (2014) ncPred: ncRNA-Disease Association Prediction through Tripartite Network-Based Inference. Frontiers in Bioengineering and Biotechnology 2. http://dx.doi.org/10.3389/fbioe.2014.00071

2. Bauer-Mehren A, Rautschka M, Sanz F, Furlong LI (2010) DisGeNET: a Cytoscape plugin to visualize, integrate, search and analyze gene-disease networks. Bioinformatics 26 (22):29242926. http://dx.doi.org/10.1093/bioinformatics/btq538

3. Chen G, Wang Z, Wang D, Qiu C, Liu M et al. (2013) LncRNADisease: a database for long-non-coding RNA-associated diseases. Nucleic Acids Research 41 (Database issue):983-986. http://dx.doi.org/10.1093/nar/gks1099

4. Helwak A, Kudla G, Dudnakova T, Tollervey D (2013) Mapping the human miRNA interactome by CLASH reveals frequent noncanonical binding. Cell 153 (3):654-665. http://dx.doi. org/10.1016/j.cell.2013.03.043

5. Jiang $\mathrm{Q}$, Wang Y, Hao Y, Juan L, Teng Met al. (2009) miR2disease: a manually curated database for microRNA deregulation in human disease. Nucleic Acids Research 37 (Database issue):98-104 http://dx.doi.org/10.1093/nar/gkn714

6. Lekka E, Hall J (2018) Noncoding RNAs in disease. FEBS Letters 592 (17):2884-2900. http://dx.doi.org/10.1002/1873-3468.13182

7. Pio G, Ceci M, D’Elia D, Loglisci C, Malerba D (2013) A Novel Biclustering Algorithm for the Discovery of Meaningful Biological Correlations between microRNAs and their Target Genes. BMC Bioinformatics 14 (Suppl 7):8. http://dx.doi.org/10.1186/14712105-14-S7-S8

8. Wang P, Guo Q, Gao Y, Zhi H, Zhang Y et al. (2016) Improved method for prioritization of disease associated lncRNAs based on ceRNA theory and functional genomics data. Oncotarget 8 (3):4642-4655. http://dx.doi.org/10.18632/oncotarget.13964

9. Yang X, Gao L, Guo X, Shi X, Wu H et al. (2014) A Network Based Method for Analysis of lncRNA-Disease Associations and Prediction of IncRNAs Implicated in Diseases. Plos One 9 (1):e87797. http://dx.doi.org/10.1371/journal.pone.0087797. 